Gut, 1988, 29, 275-278

Alimentary tract and pancreas

\title{
Does measurement of yield pressure at the cardia during endoscopy provide information on the function of the lower oesophageal sphincter mechanism?
}

\author{
R C M McGouran, J M GAlloway, D S SPENCE, C P MORTON, \\ AND DIANNE MARCHANT
}

From the Department of Gastroenterology, Queen Elizabeth Hospital, Kings Lynn, Norfolk

SUMMARY We describe a technique for measuring the yield pressure at the cardia during upper gastrointestinal endoscopy. The test was applied to 47 patients with macroscopic oesophagitis and nine patients with achalasia. Controls were 123 patients from the routine endoscopy list in whom no abnormality was found and 21 healthy volunteers. Yield pressure was similar in both control groups, but was significantly higher in achalasia $(p<0.001)$ and lower in oesophagitis $(p<0 \cdot 001)$. Yield pressures fell dramatically after pneumatic dilatation in the achalasia patients. The test was reproducible and yield pressure was not affected by age, sex, weight, or sedation. Measurement of yield pressure during endoscopy provides information which may reflect the function of the whole antireflux mechanism, and not just the lower oesophageal sphincter.

The discovery of a high pressure zone in the lower oesophagus has focused attention on this as the main factor in preventing reflux but there is a poor correlation between lower oesophageal sphincter pressure and reflux suggesting that other factors are also important. ${ }^{1}$ There is evidence that these include the length of intra-abdominal oesophagus ${ }^{23}$ and older studies suggest that the anatomical fundus ${ }^{4}$ and the sling fibres of the stomach ${ }^{5}$ may also play a role. Yield pressure refers to the intragastric pressure needed to force the cardia open and should represent the combined effect of these.

Yield pressure has previously been studied in dogs at laparotomy ${ }^{s}$ but has not apparently been studied at endoscopy in man. The purpose of this study was first to devise a reproducible method for measuring the intragastric pressure at which the cardia opened during air insufflation at endoscopy and secondly, as the presence of the endoscope traversing the cardia might influence the results, to determine if yield pressure measurements taken in this way reflected the function of the antireflux mechanism. To this end patients with macroscopic oesophagitis and thus clearly incompetent sphincters were compared with

Address for correspondence: Dr R C M McGouran, Medical Dept, Queen Elizabeth Hospital, Gayton Road, King's Lynn, Norfolk.

Received for publication 24 August 1987. achalasia patients with hypertensive sphincters, and two control groups.

\section{Methods}

PATIENTS

Patients were taken from the routine endoscopy list. Those with abnormal endoscopies other than with oesophagitis, or achalasia were excluded from the study. The presence of a hiatus hernia was noted but did not exclude patients. To avoid subjective bias oesophagitis was diagnosed only when linear streaking or frank ulceration was present. Patients were divided into three groups; those with macroscopic oesophagitis (47), those with achalasia (confirmed by barium studies) (nine) and a control group of those with no abnormality at endoscopy (123). A second control group comprised 21 healthy volunteers with no history of upper GI symptoms. Patients' ages ranged from 21-79 years (mean 53) and healthy volunteers ages from 17-57 years (mean 32). Patient's age and weight and the sedative dosages used were noted at the time of endoscopy. Reproducibility was assessed by repeating the test at the end of the endoscopy examination in 34 subjects. In five patients with achalasia yield pressure measurements were repeated once after pneumatic dilatation and in one patient twice. 
Fig. 1 Retroverted view of cardia (a) at start of air insufflation and $(b)$ at end point when cardia opens $(O P)$. The cardia opens suddenly and the end point is easily identified and often accompanied by a passive burp of air.

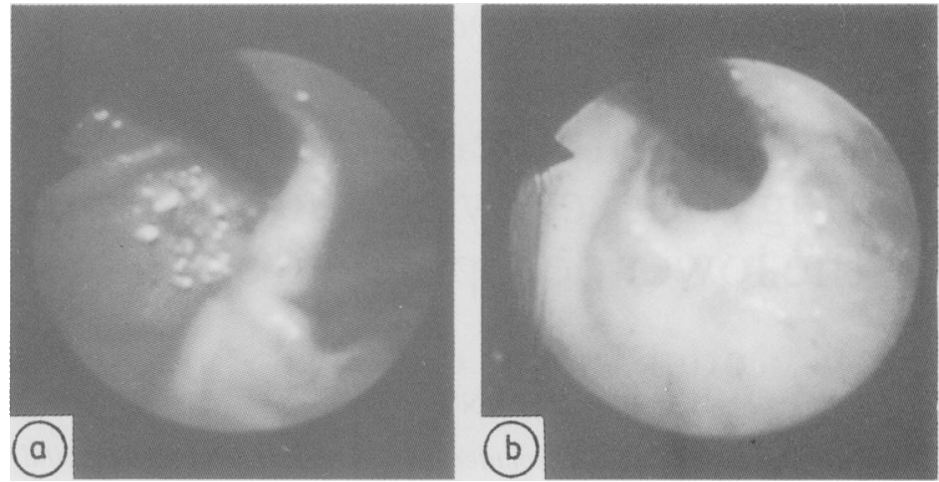

Two endoscopists were involved in the study and a small diameter endoscope (Olympus P3) was used for all examinations. Intragastric pressure was recorded through a fluid filled catheter passed through the biopsy channel of the endoscope and connected to a disposable transducer (Gould Medical Ltd). Pressure measurements were recorded on paper by standard recording equipment (Roche Bioelectronics).

Yield pressure was measured as follows. A lignocaine throat spray was used and the patient sedated with diazepam and pethidine until drowsy. The endoscope was passed into the stomach under direct vision insufflating as little air as possible. Air in the stomach was aspirated and, with the tip of the endoscope in the pyloric antrum, the catheter was passed down the biopsy channel so that a few centimetres protruded into the stomach. The resting gastric pressure (RGP) was recorded and the catheter retracted into the endoscope. Resting gastric pressure is the same throughout the stomach but the antrum is technically the easiest place to record it as the small amount of air that remains here after aspiration allows visualisation of the catheter tip. The tip of the endoscope was then retroverted and the instrument withdrawn so that the cardia could be visualised from below. With the catheter once more passed into the stomach air was insufflated until rising intragastric pressure forced the cardia open (Fig. 1). The presence of a hiatus hernia does not interfere with this observation. The opening pressure at this point was recorded (OP) and the yield pressure of the cardia (YP) calculated as OP-RGP.

STATISTICAL ANALYSIS

For normally distributed data differences between groups were analysed using Student's two-tailed unpaired $t$ test and for non-normally distributed data Wilcoxon's rank-sum-tests. 


\section{Results}

Mean yield pressure was $21.33 \mathrm{mmHg}$ in the achalasia patients, $3.04 \mathrm{mmHg}$ in patients with oesophagitis, $7.47 \mathrm{mmHg}$ in patients with a normal endoscopy and $8.95 \mathrm{mmHg}$ in normal controls (Fig. $2)$. Both control groups were significantly different from the achalasia group $(p<0 \cdot 001)$ and from the oesophagitis group $(p<0.001)$ and there was no difference between the two control groups.

Twenty three patients had an unequivocal hiatus hernia at endoscopy. Twenty of these had oesophagitis with low yield pressures. Yield pressure in the three without oesophagitis was 3,5 , and $8 \mathrm{mmHg}$.

Five patients had repeat yield pressure studies a few weeks after pneumatic dilatation for achalasia when yield pressure had fallen to a mean of 1.80 $\mathrm{mmHg}$. In a sixth patient yield pressure did not fall after the first dilatation but fell to zero after a second successful attempt (Fig. 3).

All patients had $50 \mathrm{mg}$ of pethidine iv and the

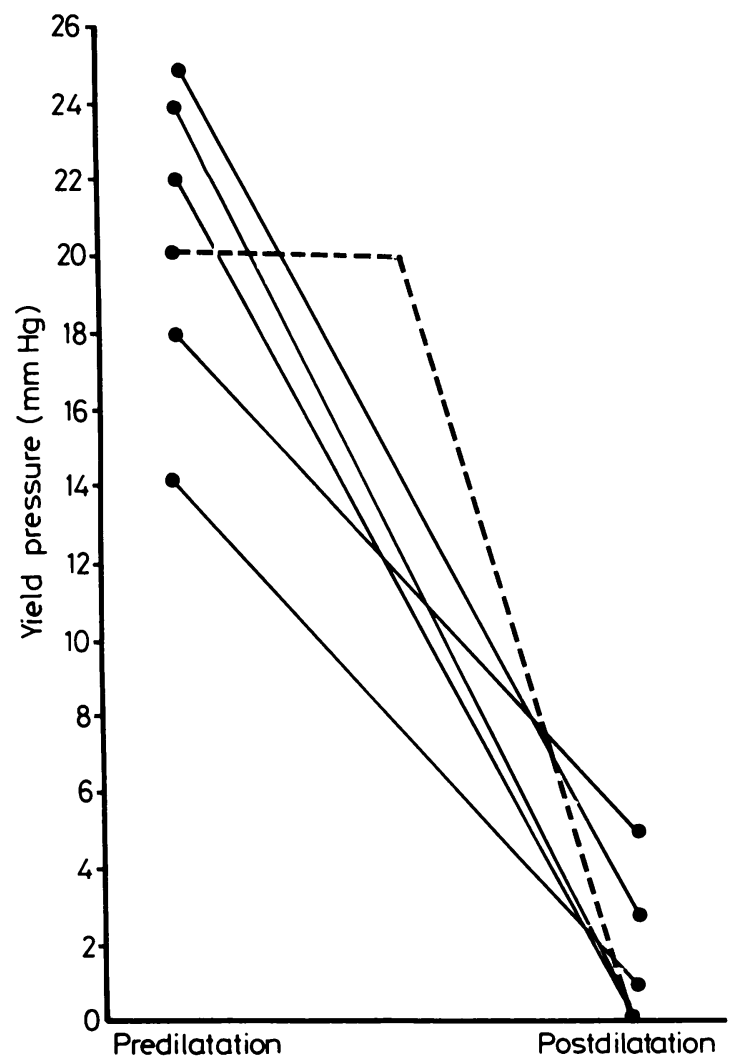

Fig. 3 Yield pressure in achalasia patients before and after pneumatic dilatation. Dotted line represents one patient who was dilated twice.

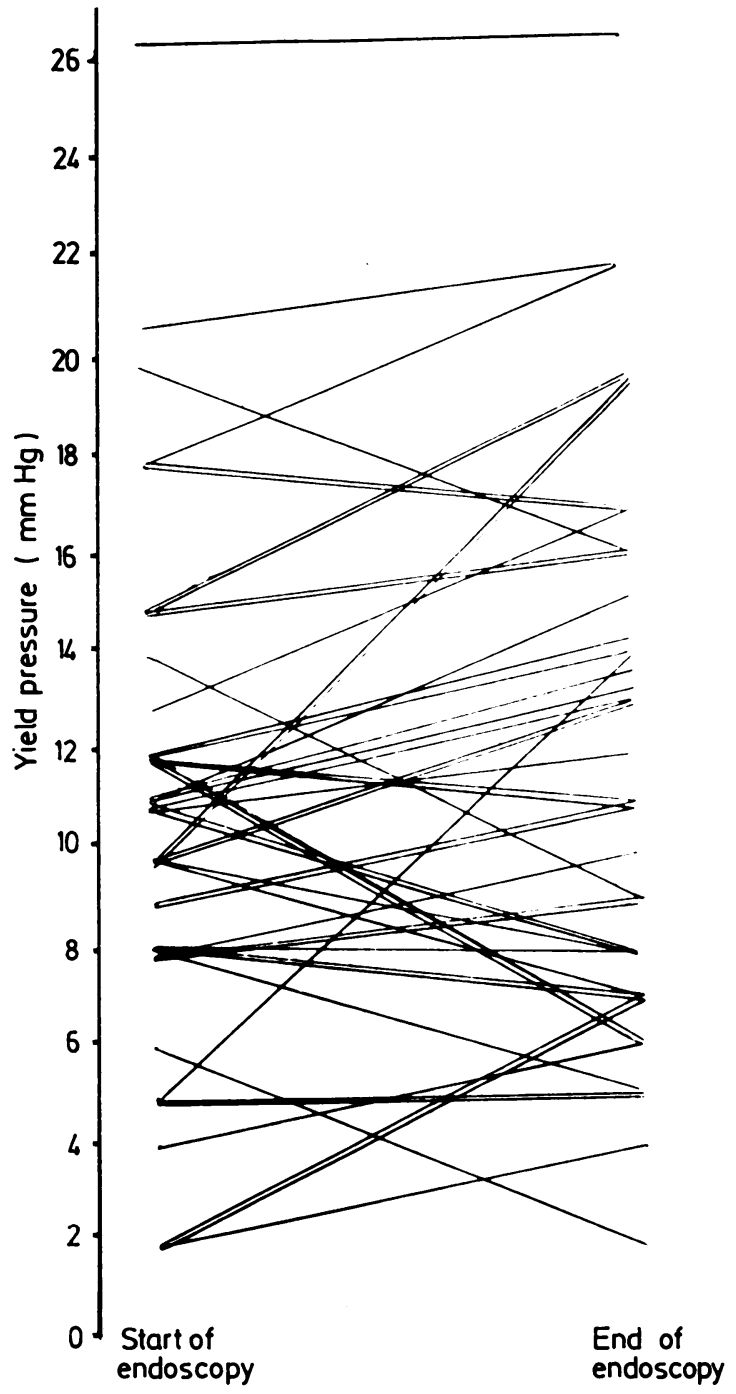

Fig. 4 Yield pressure at start and end of endoscopy in 34 patients.

diazepam dosage varied from 2.5 to $30 \mathrm{mg}$ iv. There was no correlation between sedative dosage and yield pressure.

There was no correlation between weight and yield pressure, or sex and yield pressure. The mean age of patient controls (51) was significantly lower than patients with oesophagitis $(58)(p<0.01)$ but no overall correlation was detected between yield pressure and age.

In the 34 subjects in whom reproducibility was assessed yield pressure varied between the beginning and the end of the endoscopy examination by a mean of $2.4 \mathrm{mmHg}$ (Fig. 4). 


\section{Discussion}

It is $\mathbf{3 0}$ years since the high pressure zone at the cardia was first identified manometrically ${ }^{67}$ and since then attention has focused on its role in preventing oesophageal reflux. It is unlikely, however, that such an important physiological function would be vested solely in such a weak sphincter and other mechanisms must contribute to competence at the cardia. These probably include the effect of intraabdominal pressure on the lower oesophagus, ${ }^{23}$ the anatomical arrangement of the fundus ${ }^{4}$ and the sling fibres of the stomach. ${ }^{5}$ Yield pressure refers to the intragastric pressure needed to force the cardia open and may represent the combined effects of all the antireflux mechanisms.

We were naturally concerned that the endoscope traversing the cardia would affect the results and for this reason we chose to carry out the test on patients with gross abnormalities of the antireflux mechanism. The striking difference in mean yield pressure between achalasia, oesophagitis and control groups confirms the validity of the test as a measure of the efficiency of this mechanism. In achalasia the high yield pressure is presumably a product solely of the lower oesophageal sphincter whereas in oesophagitis the low pressures may represent failure of any or all of the factors mentioned above.

Reproducibility of the test was satisfactory, although in some patients there were wide differences between yield pressures at the beginning and end of the endoscopy. Once the cardia has been forced open it takes a few minutes for its tone to return $^{5}$ and if the test is repeated too soon the second reading may be misleadingly low. Similarly if the patient retches during intubation the initial reading may be incorrect.

The majority of patients with a hiatus hernia had oesophagitis and low yield pressures. We did not identify sufficient patients with a hernia without oesophagitis to allow us to determine if the hernia itself causes a low yield pressure.

In clinical practice we have found yield pressure measurements unhelpful in detecting significant reflux because of the number of controls with low pressures. Some of these may in fact be refluxers without oesophagitis and our strict definition of macroscopic oesophagitis will have placed some refluxers in the control group. We are therefore studying the relationship between yield pressure and reflux as determined by 24 hour $\mathrm{pH}$ monitoring.

This test has particular application as a research tool, allowing as it does an assessment of the combined effects of all the factors preventing reflux and we are currently using it to study the mechanism of action of antireflux surgery.

\section{References}

1 Katz PO, Castell DO. Esophageal manometry. In: Castell DO, Wu WC, Ott DJ, eds. Gastrooesophageal reflux disease. New York: Futura Publishing, 1985: 129-38.

2 DeMeester TR, Wernley JA, Bryant GH, Little AG, Skinner DB. Clinical and in vitro determinants of gastroesophageal competence: a study of the principles of antireflux surgery. Am J Surg 1979; 137: 39-46.

3 O'Sullivan GC, DeMeester TR, Joelsson BE, et al. Interaction of lower oesophageal sphincter pressure and length of sphincter in the abdomen as determinants of gastroesophageal competence. Am J Surg 1982; 143: 40-7.

4 Marchand $P$. The gastrooesophageal sphincter and the mechanism of regurgitation. Br J Surg 1955; 42: 504-13.

5 Smiddy FG, Atkinson M. Mechanisms preventing gastrooesophageal reflux in the dog. BrJ Surg 1960; 47: 680-7.

6 Fyke FE, Code CF, Schlegel JF. The gastrooesophageal sphincter in healthy human beings. Gastroenterologia (Basel) 1956; 86: 135-50.

7 Atkinson M, Edwards DAW, Honor AJ, Rowlands EN. Comparison of cardiac and pyloric sphincters: a manometric study. Lancet 1957; ii: 918-22. 